\title{
Evaluation of risk factors of vaginal cuff dehiscence after hysterectomy
}

Myung Ji Kim', Seongmin Kim², Hyo Sook Bae², Jae Kwan Lee ${ }^{3}$ Nak Woo Lee, Jae Yun Song²

${ }^{1}$ Korea University School of Medicine, Seoul; Department of Obstetrics and Gynecology, ${ }^{2}$ Korea University Anam Hospital, Seoul, ${ }^{3}$ Korea University Guro Hospital, Seoul, ${ }^{4}$ Korea University Ansan Hospital, Ansan, Korea

\section{Objective}

The purpose of this study was to evaluate risk factors of vaginal cuff dehiscence or evisceration according to the type of operation.

\section{Methods}

Medical records of 604 women who underwent hysterectomies at Korea University Anam Hospital between June 2007 and June 2011 were reviewed. They were allocated to six groups. The six types of hysterectomies included robotic hysterectomy $(n=7)$, robotic radical hysterectomy and node dissection (RRHND, $n=9$ ), total laparoscopic hysterectomy $(T L H, n=274)$, laparoscopy assisted vaginal hysterectomy $(L A V H, n=238)$, laparoscopic radical hysterectomy and node dissection $(n=11)$, and abdominal radical hysterectomy $(A R H, n=63)$. The characteristics and outcomes of each groups were compared.

\section{Results}

There was no difference in the characteristics of patients between 6 groups. In total of 604 hysterectomies, 3 evisceration $(0.49 \%)$ and 21 dehiscences (3.47\%) occurred. Evisceration were found in RRHND $(1 / 9,11.1 \%)$, TLH (1/276, $0.36 \%)$, and ARH $(1 / 63,1.56 \%)$. Dehiscences occurred in TLH $(15 / 274,5.42 \%)$, LAVH $(4 / 238,1.68 \%)$, and ARH $(2 / 63$, $3.17 \%)$. In 169 cases of TLH with intra-corporeal continuous suture, 1 evisceration and 4 dehiscences occurred, whereas 11 dehiscences occurred in $105 \mathrm{TLH}$ cases with vaginal continuous locking suture $(2.96 \%$ vs. $10.47 \%, P=0.02)$.

\section{Conclusion}

The incidence of vaginal cuff dehiscenceand eviscerationwas significantly higher in TLH than LAVH. The intra-corporeal cuff suture was superior to the vaginal suture to prevent the vaginal cuff complications in TLH.

Keywords: Complications; Hysterectomy; Surgical wound dehiscence; Suture techniques

\section{Introduction}

Hysterectomy is one of the most common gynecological surgeries performed in Korea. A variety of complication can occur with hysterectomies including bladder injury, ureteral injury, gastrointestinal injury, hemorrhage, postoperative fever, and vaginal vault prolapsed [1]. Vaginal cuff dehiscence or evisceration (VCDE) are rare but serious complication of hysterectomy. Vaginal cuff dehiscence has been defined as a full thickness separation, partial or total, of the anterior and posterior edges of the vaginal cuff with or without bowel evisceration [2]. The distal ileum is the most frequent eviscerating organ when vaginal evisceration is observed although prolapse of
Received: 2013.4.25. Revised: 2013.9.26. Accepted: 2013.10.20.

Corresponding author: Jae Yun Song

Department of Obstetrics and Gynecology, Korea University Anam Hospital, 73 Inchon-ro, Seongbuk-gu, Seoul 136-705, Korea Tel: +82-2-920-6775 Fax: +82-2-921-5357

E-mail: sjyuni105@gmail.com

Articles published in Obstet Gynecol Sci are open-access, distributed under the terms of the Creative Commons Attribution Non-Commercial License (http://creativecommons. org/licenses/by-nc/3.0/) which permits unrestricted non-commercial use, distribution, and reproduction in any medium, provided the original work is properly cited.

Copyright $\odot 2014$ Korean Society of Obstetrics and Gynecology 


\section{Obstetrics \& Gynecology Science}

Myung Ji Kim, et al. Cuff dehiscence after hysterectomy

the omentum, appendix and fallopian tubes have also been reported [3]. When it occurs, prompt surgical and medical intervention is required to ensure optimal care.

Hysterectomy can now be performed utilizing minimally invasive surgical techniques such as laparoscopy. Previous studies suggest that the rate of VCDE seems to have been shown to increase after laparoscopic surgeries compared with that after the open approach [3-5]. The report postulated that the use of thermal energy in addition to other factors unique to laparoscopic surgery may be responsible for and increased risk of vaginal cuff dehiscence compared with other modes of total hysterectomy. As surgical equipment and techniques continue to improve, hysterectomies are increasingly performed with laparoscopy and related complications have been decreased. Possibly owing to this improvement, recently several authors have reported different surgical approaches between abdominal or vaginal and laparoscopic without substantial differences. laco et al. [6] reported that although the incidence of dehiscence is highest among total laparoscopic hysterectomies, it is not as high as previously reported. As a way of minimally invasive surgical techniques, robotic surgery is becoming common practice for hysterectomy recently. Kho et al. [7] revealed that robotic hysterectomy is feasible with minimal complications noted through the 6-week postoperative recovery time. But, in another report comparing robotic surgery to other mode of operation, the robotic surgery showed worse postoperative prognosis [8].

Not only surgical modality but also type of hysterectomy affects the incidence of vaginal cuff complication. Nick et al. [9] reported that radical hysterectomy was associated with a 9-fold increase in vaginal cuff complication. Also, special consideration should be given to the colpotomy incision and vault closure concerning vaginal dehiscence during total laparoscopic hysterectomy (TLH) [10]. Concerning about the technical difficulty of laparoscopic surgery, this study provides suggestions for prevention of vaginal cuff problems in terms of the type of suture techniques during TLH.

This study compared the incidence of vaginal cuff problems from different approaches to hysterectomy to assess which mode of hysterectomy poses a lesser risk for vaginal cuff problems and described the patient characteristics of those with VCDE after hysterectomy to identify potential risk factors for this complication. This study has great significance because of the fact that there is a dearth of well-conducted studies on vaginal cuff dehiscence after hysterectomy in Korea.

\section{Materials and methods}

We retrospectively analyzed medical reviews of 604 women who underwent hysterectomies at Korea University Anam Hospital in the period of June 2007 until June 2011. Vaginal cuff dehiscence was defined as partial or total full thickness opening of the anterior and posterior edges of vaginal cuff without protruding bowels. Vaginal cuff evisceration was defined as separation with protruding bowel [2].

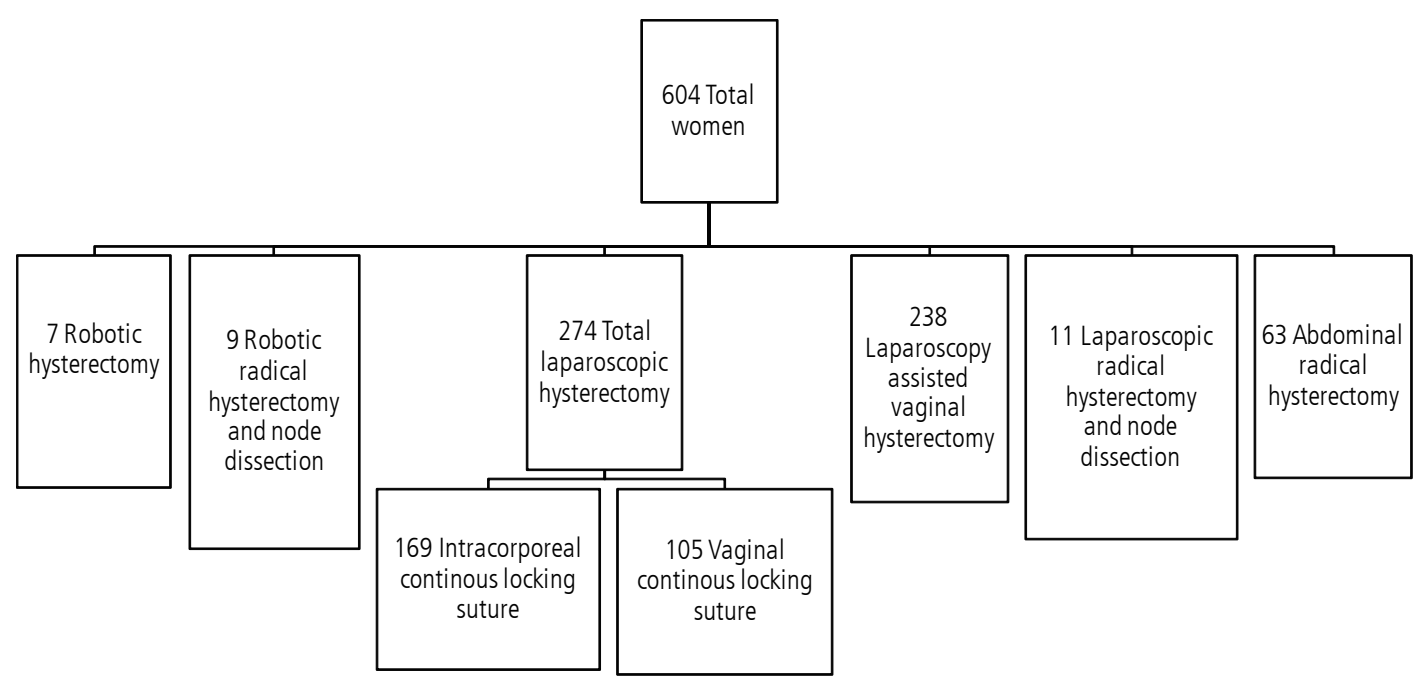

Fig. 1. Flowchart of the trial population. 


\title{
Obstetrics \& Gynecology Science
}

\author{
Vol. 57, No. 2, 2014
}

Data extracted from the medical record included patient's age, body mass index (BMI), obstetrical history and indication for surgery. Operative reports were reviewed and operative times, estimated blood loss (EBL) and techniques of vaginal cuff closure were recorded. EBL was estimated by the gap between the amount of the fluid suctioned and that used on irrigation in laparoscopic or robotic surgery. In the cases of laparotomy, we counted the number of used gauzes to calculate the EBL. Postoperative records were also reviewed. These included postoperative fever, period of hospitalization, transfusion, hemoglobin $(\mathrm{Hb})$, white blood cell $(\mathrm{WBC})$, erythrocyte sedimentation rate, C-reactive protein, use of antibiotics and post-op complications other than VCDE. We also collected additional data from those who developed VCDE including history of operation, stage, lympho-vascular invasion and resection margin.

The main details considered for the analysis were surgical methods and suture techniques. A total of 604 women were allocated to six groups. The six types of hysterectomies included robotic hysterectomy $(n=7)$, robotic radical hysterectomy and node dissection (RRHND, $n=9), T L H(n=274)$, laparoscopy assisted vaginal hysterectomy ( $L A V H, n=238$ ), laparoscopic radical hysterectomy and node dissection (LRHND, $n=11)$, and abdominal radical hysterectomy (ARH, $n=63$ ) (Fig. 1). The da Vinci Surgical System has been used for robotic hysterectomy at our institution.

Three subcategories of laparoscopic hysterectomy (LH) have been described [11]. In LAVH, the procedure is done partially with laparoscopy and partly vaginally, but the laparoscopic component does not involve uterine vessel ligation. In uterine vessel ligation $\mathrm{LH}$, although the uterine vessels are ligated with laparoscopy, part of the operation is donevaginally. In our study, including node dissection, LH is subclassified as LRHND. In TLH, the entire operation (including suture of the vaginal vault) is performed with laparoscopy.

In patients undergoing TLH, two methods for vaginal cuff closure were used, either intracorporeal continuous suture $169(61 \%)$ or vaginal continuous locking suture 105 (38\%) according to the single surgeon's decision (Fig. 1). Only intracorporeal continuous suture was performed in robotic surgery, while vaginal continuous suture was performed in LAVH. Five-point suture with figure of eight was performed in total abdominal hysterectomy. Subanalysis included age, BMI, operation time, parity, EBL, postoperative fever, and use of postoperative antibiotics.

Data were analyzed using SPSS ver. 15.0 (SPSS Inc., Chicago, IL, USA), with a significance set at $P<0.05$. Comparisons of baseline characteristics between groups were drawn using ANOVA test. Two different vaginal cuff suture techniques used in hysterectomy were compared with two tailed chi-square test. Associations between categorical variables and VCDE were determined using Kruskal-Wallis test.

\section{Results}

The baseline characteristics were shown in Table 1. Age, BMI, parity, operation time, EBL, hospitalized days, initial serum $\mathrm{Hb}$

Table 1. Baseline characteristics of all participants according to operation types

\begin{tabular}{|lcccccrr|}
\hline & $\begin{array}{c}\text { RH } \\
(\mathbf{n}=\mathbf{7})\end{array}$ & $\begin{array}{c}\text { RRHND } \\
(\mathbf{n}=\mathbf{9})\end{array}$ & $\begin{array}{c}\text { TLH } \\
(\mathbf{n}=\mathbf{2 7 4})\end{array}$ & $\begin{array}{c}\text { LAVH } \\
(\mathbf{n}=\mathbf{2 3 8})\end{array}$ & \multicolumn{1}{c}{$\begin{array}{c}\text { LRHND } \\
(\mathbf{n}=\mathbf{1 1})\end{array}$} & \multicolumn{1}{c}{$\begin{array}{c}\text { ARH } \\
(\mathbf{n}=63)\end{array}$} & $\boldsymbol{P}$-value \\
\hline Age $(\mathrm{yr})$ & $43.6 \pm 11.1$ & $54.4 \pm 2.6$ & $48.5 \pm 6.5$ & $49.1 \pm 5.9$ & $53.4 \pm 12.0$ & $54.4 \pm 2.6$ & $<0.01$ \\
BMI $\left(\mathrm{kg} / \mathrm{m}^{2}\right)$ & $22.2 \pm 3.2$ & $25.7 \pm 5.2$ & $24.2 \pm 3.3$ & $24.0 \pm 3.7$ & $23.3 \pm 2.6$ & $23.9 \pm 4.0$ & 0.38 \\
Parity & $1.6 \pm 1.3$ & $2.3 \pm 0.8$ & $1.8 \pm 0.8$ & $1.9 \pm 0.7$ & $3.0 \pm 1.5$ & $2.0 \pm 1.5$ & 0.01 \\
Operation time (min) & $303.9 \pm 95.6$ & $372.1 \pm 69.1$ & $118.1 \pm 40.1$ & $125.3 \pm 40.6$ & $293.4 \pm 74.3$ & $188.5 \pm 78.0$ & $<0.01$ \\
EBL $(\mathrm{mL})$ & $968.9 \pm 461.2$ & $525.0 \pm 223.0$ & $552.5 \pm 275.5$ & $620.1 \pm 285.9$ & $972.7 \pm 436.1$ & $890.3 \pm 621.2$ & $<0.01$ \\
HD $($ day) & $19.2 \pm 8.1$ & $12.9 \pm 5.2$ & $6.9 \pm 3.3$ & $6.5 \pm 2.6$ & $26.7 \pm 19.0$ & $20.9 \pm 9.6$ & $<0.01$ \\
Initial Hb $(\mathrm{g} / \mathrm{dL})$ & $12.4 \pm 1.5$ & $12.2 \pm 0.6$ & $12.2 \pm 1.5$ & $12.1 \pm 1.5$ & $12.6 \pm 1.4$ & $12.2 \pm 1.3$ & 0.36 \\
Initial $\mathrm{WBC}(/ \mu \mathrm{L})$ & $6,246.6 \pm 1,320$ & $7,141.4 \pm 1,425$ & $6,716.8 \pm 2,126$ & $6,659.2 \pm 2,159$ & $2,886.3 \pm 1,197$ & $7,683.5 \pm 711$ & 0.24 \\
\hline
\end{tabular}

Values are presented as mean \pm standard deviation except $P$-values.

$\mathrm{RH}$, robotic hysterectomy; RRHND, robotic radical hysterectomy and node dissection; TLH, total laparoscopic hysterectomy; LAVH, laparoscopy assisted vaginal hysterectomy; LRHND, laparoscopic radical hysterectomy and node dissection; ARH, abdominal radical hysterectomy; BMI, body mass index; EBL, estimated blood loss; HD, hospitalized days; Hb, hemoglobin; WBC, white blood cell. 


\section{Obstetrics \& Gynecology Science}

Myung Ji Kim, et al. Cuff dehiscence after hysterectomy

Table 2. Incidence of vaginal cuff complications by mode of hysterectomy

\begin{tabular}{|lccc|}
\hline Operation type & Total cases & Dehiscence & Evisceration \\
\hline Robotic hysterectomy & 7 & $0(0)$ & $0(0)$ \\
Robotic radical hysterectomy and node dissection & 9 & $10)$ & $1(11.1)$ \\
Total laparoscopic hysterectomy & 274 & $4(5.43)$ & $1(0.36)$ \\
Laparoscopy assisted vaginal hysterectomy & 238 & $0(0)$ & $0(0)$ \\
Laparoscopic radical hysterectomy and node dissection & 11 & $2(3.17)$ & $1(1.56)$ \\
Abdominal radical hysterectomy & 63 & $21(3.47)$ & $3(0.49)$ \\
Total & 604 & & 0 \\
\hline
\end{tabular}

Values are presented as number or number (\%).

Table 3. Clinical data of the patients complicated by vaginal cuff problems

\begin{tabular}{|c|c|c|c|c|c|c|}
\hline Patient & Age (yr) & BMI $\left(\mathrm{kg} / \mathrm{m}^{2}\right)$ & Parity $^{\text {a) }}$ & Indication & Comorbidity & Evisceration \\
\hline 1 & 49 & 24.0 & $\mathrm{G} 2 \mathrm{P} 2$ & Cervical cancer & No & Yes \\
\hline 2 & 50 & 20.7 & G4P2 & Uterine leiomyoma & No & No \\
\hline 3 & 52 & 23.3 & G5P3 & Uterine leiomyoma & No & No \\
\hline 4 & 37 & 20.1 & G3P2 & Uterine leiomyoma & No & No \\
\hline 5 & 46 & 18.4 & G3P2 & Uterine leiomyoma & Right Bartholin cyst & No \\
\hline 6 & 43 & 29.7 & G5P1 & Uterine leiomyoma & No & No \\
\hline 7 & 45 & 36.4 & G4P2 & $\mathrm{CIS}$ & No & No \\
\hline 8 & 50 & 25.8 & G1P0 & Uterine leiomyoma & Left ovarian cyst & No \\
\hline 9 & 45 & 26.1 & G4P2 & Uterine leiomyoma & No & No \\
\hline 10 & 48 & 23.7 & $\mathrm{G} 2 \mathrm{P} 2$ & Uterine leiomyoma & No & No \\
\hline 11 & 40 & 22.4 & G5P2 & Uterine leiomyoma & No & No \\
\hline 12 & 51 & 21.8 & G6P1 & Uterine leiomyoma & Adenomyosis, PID & No \\
\hline 13 & 52 & 21.1 & G4P2 & Endometrial hyperplasia & No & No \\
\hline 14 & 49 & 20.4 & G7P1 & $\mathrm{CIS}$ & No & No \\
\hline 15 & 50 & 28.9 & G2P1 & Uterine leiomyoma & No & No \\
\hline 16 & 51 & 26.4 & G4P2 & Severe dysplasia & No & No \\
\hline 17 & 40 & 20.9 & G3P1 & Uterine leiomyoma & No & Yes \\
\hline 18 & 55 & 29.8 & G3P2 & Uterine leiomyoma & No & No \\
\hline 19 & 52 & 22.2 & G1P1 & Uterine leiomyoma & No & No \\
\hline 20 & 53 & 22.6 & G5P3 & Adenomyosis & No & No \\
\hline 21 & 48 & 22.2 & $\mathrm{G} 2 \mathrm{P} 2$ & Uterine leiomyoma & No & No \\
\hline 22 & 50 & 22.2 & G2P2 & Cervical cancer & No & No \\
\hline 23 & 56 & 19.2 & GOPO & Ovarian cancer & No & Yes \\
\hline 24 & 50 & 24.4 & G4P2 & Endometrial cancer & No & No \\
\hline
\end{tabular}

BMI, body mass index; CIS, squamous cell carcinoma in situ of cervix; PID, pelvic inflammatory disease.

a)Parity was shown with G (gravida) and P (para) .

and WBC level were compared between 6 different groups according to the operation type. There was some heterogeneity between groups in age, parity, operation time, EBL, hospitalized days ( $P$-value was shown in Table 1$)$. BMI, initial serum
$\mathrm{Hb}$ and WBC were not significantly different between groups ( $P=0.38, P=0.36, P=0.24$, respectively).

In total of 604 hysterectomies, 3 eviscerations $(0.49 \%)$ and 21 dehiscences (3.47\%) occurred. Eviscerations were found 


\section{Obstetrics \& Gynecology Science}

Vol. 57, No. 2, 2014

Table 4. Surgical data of the patients complicated by vaginal cuff problems

\begin{tabular}{|c|c|c|c|c|c|c|c|}
\hline Patient & $\begin{array}{c}\text { Mode of } \\
\text { hysterectomy }\end{array}$ & Additional procedure & $\begin{array}{l}\text { Operation } \\
\text { time (min) }\end{array}$ & $\begin{array}{l}\text { EBL } \\
(\mathrm{mL})\end{array}$ & Fever $^{\text {a) }}$ & $\begin{array}{l}\text { Hospital stay } \\
\text { (day) }\end{array}$ & $\begin{array}{c}\text { Use of } \\
\text { antibiotics }^{\text {b) }}\end{array}$ \\
\hline 1) & RRHND & None & 325 & 800 & 2 & 33 & 2 \\
\hline 2 & TLH & None & 73 & 500 & 0 & 14 & 1 \\
\hline 3 & TLH & None & 100 & 500 & 0 & 7 & 0 \\
\hline 4 & TLH & None & 100 & - & 0 & 7 & 0 \\
\hline 5 & TLH & Right Bartholin cystectomy & 150 & - & 0 & 6 & 0 \\
\hline 6 & TLH & None & 185 & 500 & 1 & 7 & 0 \\
\hline 7 & TLH & None & 95 & 100 & 0 & 8 & 0 \\
\hline 8 & TLH & LSO & 110 & - & 0 & 9 & 0 \\
\hline 9 & TLH & None & 98 & - & 0 & 5 & 0 \\
\hline 10 & TLH & None & 145 & - & 0 & 5 & 0 \\
\hline 11 & TLH & None & 80 & 500 & 0 & 6 & 0 \\
\hline 12 & TLH & None & 190 & 600 & 0 & 12 & 0 \\
\hline 13 & TLH & BSO & 65 & 500 & 0 & 7 & 0 \\
\hline 14 & TLH & None & 70 & 500 & 0 & 7 & 0 \\
\hline 15 & TLH & None & 210 & 1,000 & 0 & 9 & 1 \\
\hline 16 & TLH & None & 100 & 500 & 1 & 6 & 0 \\
\hline $17^{c)}$ & TLH & None & 65 & - & 0 & 6 & 0 \\
\hline 18 & LAVH & $\mathrm{BSO}$ & 100 & 100 & 1 & 5 & 0 \\
\hline 19 & LAVH & BSO adhesiolysis & 180 & 180 & 0 & 4 & 1 \\
\hline 20 & LAVH & Appendectomy & 105 & 105 & 0 & 4 & 0 \\
\hline 21 & LAVH & None & 90 & 90 & 0 & 5 & 0 \\
\hline 22 & ARH & $\mathrm{BSO}$ & 300 & 2,100 & 2 & 71 & 2 \\
\hline $23^{c}$ & ARH & BSO omentectomy & 260 & 800 & 2 & 17 & 1 \\
\hline 24 & ARH & $\mathrm{BSO}$ & 165 & 500 & 0 & 11 & 1 \\
\hline
\end{tabular}

EBL, estimated blood loss; RRHND, robotic radical hysterectomy and node dissection; TLH, total laparoscopic hysterectomy; LSO, left salpingo-oophorectomy; BSO, bilateral salpingo-oophorectomy; LAVH, laparoscopy assisted vaginal hysterectomy; ARH, abdominal radical hysterectomy.

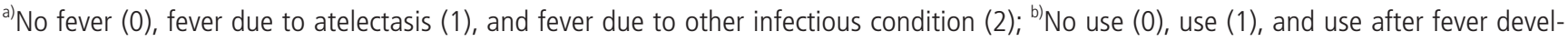
oped (2); ${ }^{c}$ Dehiscence with evisceration.

in RRHND (1/9, 11.1\%), TLH (1/274, 0.36\%), and ARH (1/64, 1.56\%). Dehiscences occurred in TLH (15/274, 5.43\%), LAVH $(4 / 238,1.68 \%)$, and ARH $(2 / 63,3.17 \%)$ (Table 2). We analyzed patient and surgical characteristics of vaginal cuff dehiscence with or without evisceration by mode of hysterectomy (Tables 3, 4).

As a subgroup analysis, we compared the characteristics and VCDE occurrence rate between the TLH and LAVH groups. Age, BMI, parity, operation time, EBL, hospitalized days, initial serum $\mathrm{Hb}$ level, postoperation day-1 serum $\mathrm{Hb}$ level, initial serum WBC level, and post-operation day-1 WBC level were not significantly different between two groups. The VCDE oc- currence rate was $5.79 \%$ in TLH group and $1.68 \%$ in LAVH group, and it was significantly higher in TLH group ( $P=0.016)$. In the cases of TLH, there was a significant difference in vaginal cuff problems depending on the suture method of vaginal cuff (Table 5). In 169 cases of TLH with intracorporeal continuous suture, 1 evisceration and 4 dehiscences (2.96\%) occurred, whereas 11 dehiscences (10.47\%) occurred in 105 TLH cases with vaginal continuous locking suture. Durations between the day of operation and VCDE in the cases of intracorporeal suture and vaginal suture were 72.8 and 23.6 days respectively, and it took significantly longer time in intracorporeal suture than in vaginal suture $(P=0.01)$. 


\section{Obstetrics \& Gynecology Science}

Myung Ji Kim, et al. Cuff dehiscence after hysterectomy

Table 5. Comparison of two different vaginal cuff suture techniques used in total laparoscopic hysterectomy

\begin{tabular}{|lcccc|}
\hline \multicolumn{1}{|c}{ Suture method } & Total cases & Dehiscence & Evisceration & Total VCDE \\
\hline Intracorporeal $^{\text {a) }}$ & 169 & 4 & 1 & $5(2.63 \%)$ \\
Vaginal $^{\text {b) }}$ & 105 & 11 & 0 & $11(10.47 \%)$ \\
Total & 274 & 15 & 1 & $16(5.38 \%)$ \\
\hline
\end{tabular}

The intracorporeal continuous suture was superior than vaginal continuous locking suture in prevention of vaginal cuff complications $(P=0.02)$.

VCDE, vaginal cuff dehiscence or evisceration.

${ }^{a)}$ Intracorporeal continuous suture; ${ }^{\text {b)}}$ Vaginal approach continuous locking suture.

Table 6. Evaluation of risk factors for vaginal cuff problems

\begin{tabular}{|c|c|c|c|c|}
\hline & $\begin{array}{l}\text { No complication } \\
(n=580)\end{array}$ & $\begin{array}{l}\text { Dehiscence } \\
(n=21)\end{array}$ & $\begin{array}{l}\text { Evisceration } \\
\quad(n=3)\end{array}$ & $P$-value \\
\hline Age (yr) & 49.4 & 48.4 & 48.3 & 0.99 \\
\hline BMI $\left(\mathrm{kg} / \mathrm{m}^{2}\right)$ & 24.1 & 24.2 & 21.4 & 0.32 \\
\hline Operation time (min) & 135.2 & 129.1 & 216.7 & 0.46 \\
\hline $\mathrm{EBL}(\mathrm{mL})$ & 652.3 & 643.8 & 800.0 & 0.32 \\
\hline Fever $^{\mathrm{a})}$ & 0.26 & 0.24 & 1.33 & 0.06 \\
\hline Use of antibiotics ${ }^{\mathrm{b})}$ & 0.26 & 0.21 & 0.50 & 0.42 \\
\hline
\end{tabular}

BMI, body mass index; EBL, estimated blood loss.

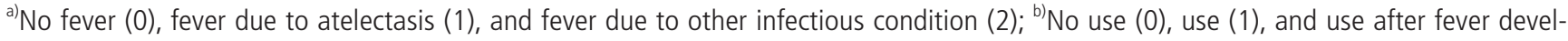
oped (2).

Subanalysis was performed to analyze the risk factors associated with VCDE, including age, BMI, operation time, EBL, postoperative fever, use of postoperative antibiotics, and none of these factors had correlation with VCDE (Table 6).

\section{Discussion}

Vaginal cuff dehiscence after hysterectomy is a rare, but potentially devastating complication. If it is not corrected in a rapid fashion, there is significant potential for morbidity and mortality. The incidence of this condition is not clear, ranging in the literature between $0 \%$ and $7.5 \%$ [12]. A review of records from 1970 to 2001 at the Mayo Clinic Rochester revealed a low incidence $(0.032 \%)$ of vaginal evisceration after pelvic operation via abdominal and vaginal approach [13]. At a single, large, referral institution, TLH was associated with an increased incidence of vaginal cuff dehiscence compared with other approaches (4.93\% compared with $0.29 \%$ total vaginal hysterectomy, and $0.12 \%$ total abdominal hysterectomy) [5]. In 2006, laco et al. [6] published a $0.28 \%$ incidence of evisceration after 3,593 hysterectomies. Although a greater rate of evisceration after laparoscopic hysterectomies was reported $(0.26 \%$ abdominal, $0.25 \%$ vaginal, and $0.79 \%$ laparoscopic), the authors concluded that route of surgery does not influence risk of dehiscence because the difference was not statistically significant. In this study, the overall incidence of dehiscence without and with evisceration was 3.47\% and $0.49 \%$ respectively. The overall incidence is low, but not negligible; therefore, the diagnosis and therapy of this posthysterectomy complication must be kept in mind. However, it should be noted that this study is limited by the small number of robotic hysterectomies and only three vaginal evisceration was reported among RRHND, TLH, and ARH together (one case by each). In subgroup analysis, the VCDE occurrence rate was significantly higher in TLH group than LAVH group. This is supported by several previous studies $[4,5]$.

We also hypothesized that the vaginal cuff suture method could influence the occurrence of VCDE. In this study, there was a significant difference in vaginal cuff problems depending on the suture method of vaginal cuff. Intracorporeal continuous suture was superior to vaginal approach continuous locking suture in TLH performed in our institution. This result could be regarded ironic because of the higher VCDE incidence in TLH than in LAVH, in which the vaginal cuff is almost always closed by vaginal suture. But the cut surface of vaginal 


\title{
Obstetrics \& Gynecology Science
}

\author{
Vol. 57, No. 2, 2014
}

cuff is not similar between two surgeries. In LAVH, the cut level of vaginal cuff is very close to cervix, so the cut surface is curved toward inside the vagina. However, in TLH, the cuff is mostly made at the level of vesico-vaginal junction and rectovaginal junction, and the vaginal cuff is not so curved as in LAVH. In this point of view, the anterior and posterior cut surfaces of vaginal cuff would not attach properly with vaginal suture technique, because the closest layer between anterior and posterior surface is vaginal mucosa, not the pelvic serosa. Consequently, intracorporeal suture would give less tension to vaginal cuff than vaginal suture in cuff closure after TLH. However, Uccella et al. [8] reported that transvaginal colporrhaphy after TLH is associated with a reduction in risk of VCDE compared with laparoscopic suture, and Hwang et al. [14] found no difference between two groups. This heterogeneity between studies could be come from the difference of suture technique of the surgeons, or the development in suture materials may act as a bias. Thus, further study is needed about this correlation.

Besides surgical factors, some potential underlying patientrelated risk factors are associated with VCDE incidence. Somkuti et al. [15] described 10 risk factors for apical vaginal rupture after an abdominal or vaginal hysterectomy: poor technique, postoperative infection, hematoma, coitus before healing, age, radiotherapy, corticosteroid therapy, trauma or rape, previous vaginoplasty, and use of the Valsalva maneuver. Furthermore the causes of vaginal cuff dehiscence and evisceration differ between premenopausal and postmenopausal women. Vaginal evisceration was associated with chronic pelvic prolapse, atrophic vaginal tissue associated with a sudden increase in abdominal pressure for postmenopausal women. In the elderly, the evisceration was usually a spontaneous event and happened quite late [16]. In young patients, sexual intercourse before complete healing of the vaginal cuff is considered the main trigger event. In this group of patients, the complication had an early onset [17]. Unfortunately, we did not collect data for several postoperative triggering events that could have had an impact on the incidence of vaginal dehiscence. Although our study was not designed to determine the causes of vaginal cuff dehiscence after hysterectomy this time, we hope to address this clinically important question with follow-up prospective studies.

We acknowledge limitations of the current study. First, a significant heterogeneity in cuff complication rates was found between studies, likely attributable to variation in sample size, patient selection. Second, in our study, we have missed data of total abdominal hysterectomy cases. We recognize that these missing data could potentially have had an influence on our results. Third, the retrospective nature of this study lacks the randomization of mode of hysterectomy. Last, the rarity of the event required a large study population to properly assess the actual complication rate after stratification for surgical approach, and such a large number of cases could only be achieved with a multicenter retrospective study.

Although there are some limitation in our recent study, this study has great significance in the aspect of comparing the incidence of vaginal cuff problems from different approaches to hysterectomy including minimally invasive surgery at the same time to assess which mode of hysterectomy poses a lesser risk for vaginal cuff problems and described the surgical and the patient characteristics of those with VCDE after hysterectomy to identify potential risk factors for this complication.

In conclusion, the incidence of vaginal cuff dehiscence and evisceration was significantly higher in TLH than LAVH. The intra-corporeal cuff suture was superior to the vaginal suture to prevent the vaginal cuff complications in TLH.

\section{Conflict of interest}

No potential conflict of interest relevant to this article was reported.

\section{References}

1. Ramirez PT, Klemer DP. Vaginal evisceration after hysterectomy: a literature review. Obstet Gynecol Surv 2002;57:462-7.

2. Kho RM, AkI MN, Cornella JL, Magtibay PM, Wechter ME, Magrina JF. Incidence and characteristics of patients with vaginal cuff dehiscence after robotic procedures. Obstet Gynecol 2009;114(2 Pt 1):231-5.

3. Ceccaroni M, Berretta R, Malzoni M, Scioscia M, Roviglione $G$, Spagnolo $E$, et al. Vaginal cuff dehiscence after hysterectomy: a multicenter retrospective study. Eur J Obstet Gynecol Reprod Biol 2011;158:308-13.

4. Hur HC, Donnellan N, Mansuria S, Barber RE, Guido R, Lee T. Vaginal cuff dehiscence after different modes of hysterectomy. Obstet Gynecol 2011;118:794-801. 


\section{Obstetrics \& Gynecology Science}

Myung Ji Kim, et al. Cuff dehiscence after hysterectomy

5. Hur HC, Guido RS, Mansuria SM, Hacker MR, Sanfilippo JS, Lee TT. Incidence and patient characteristics of vaginal cuff dehiscence after different modes of hysterectomies. J Minim Invasive Gynecol 2007;14:311-7.

6. Iaco PD, Ceccaroni M, Alboni C, Roset B, Sansovini $M, D^{\prime}$ Alessandro $L$, et al. Transvaginal evisceration after hysterectomy: is vaginal cuff closure associated with a reduced risk? Eur J Obstet Gynecol Reprod Biol 2006;125:134-8.

7. Kho RM, Hilger WS, Hentz JG, Magtibay PM, Magrina JF. Robotic hysterectomy: technique and initial outcomes. Am J Obstet Gynecol 2007;197:113.e1-4.

8. Uccella S, Ghezzi F, Mariani A, Cromi A, Bogani G, Serati $M$, et al. Vaginal cuff closure after minimally invasive hysterectomy: our experience and systematic review of the literature. Am J Obstet Gynecol 2011;205:119.e112.

9. Nick AM, Lange J, Frumovitz M, Soliman PT, Schmeler KM, Schlumbrecht MP, et al. Rate of vaginal cuff separation following laparoscopic or robotic hysterectomy. Gynecol Oncol 2011;120:47-51.

10. Jeung IC, Baek JM, Park EK, Lee HN, Kim CJ, Park TC, et al. A prospective comparison of vaginal stump suturing techniques during total laparoscopic hysterectomy. Arch Gynecol Obstet 2010;282:631-8.

11. Reich H, Roberts L. Laparoscopic hysterectomy in current gynecological practice. Rev Gynaecol Pract 2003;3:3240.

12. DeNardis SA, Holloway RW, Bigsby GE 4th, Pikaart DP, Ahmad S, Finkler NJ. Robotically assisted laparoscopic hysterectomy versus total abdominal hysterectomy and lymphadenectomy for endometrial cancer. Gynecol Oncol 2008;111:412-7.

13. Croak AJ, Gebhart JB, Klingele CJ, Schroeder G, Lee RA, Podratz KC. Characteristics of patients with vaginal rupture and evisceration. Obstet Gynecol 2004;103:572-6.

14. Hwang JH, Lee JK, Lee NW, Lee KW. Vaginal cuff closure: a comparison between the vaginal route and laparoscopic suture in patients undergoing total laparoscopic hysterectomy. Gynecol Obstet Invest 2011;71:163-9.

15. Somkuti SG, Vieta PA, Daugherty JF, Hartley LW, Blackmon EB Jr. Transvaginal evisceration after hysterectomy in premenopausal women: a presentation of three cases. Am J Obstet Gynecol 1994;171:567-8.

16. Kowalski LD, Seski JC, Timmins PF, Kanbour Al, Kunschner AJ, Kanbour-Shakir A. Vaginal evisceration: presentation and management in postmenopausal women. J Am Coll Surg 1996;183:225-9.

17. Alessandri F, Remorgida V, Venturini PL, Ferrero S. Unidirectional barbed suture versus continuous suture with intracorporeal knots in laparoscopic myomectomy: a randomized study. J Minim Invasive Gynecol 2010;17:725-9. 